\title{
Bilateral Open Ureteroceles with Concomitant Bladder Calculi in an Adult Male
}

\author{
Ari P. Bernstein, BA, ${ }^{1}$ Michael Schulster, $M D,{ }^{2}$ Brian Chao, $M D,{ }^{2}$ and Mark V. Silva, $\mathrm{MD}^{2}$
}

\begin{abstract}
Background: Bilateral ureteroceles in adult males are a rare occurrence, made even more so with simultaneous presence of bladder calculi. Management strategies for such patients are not well documented in the literature. We present a rare case of bilateral open ureteroceles with large concomitant bladder calculi to contribute to the paucity of literature on management of such patients.

Case Presentation: We present the case of a 35-year-old man whose gross hematuria work-up demonstrated right-sided hydroureteronephrosis in the setting of bilateral ureteroceles and multiple large bladder calculi up to $3.8 \mathrm{~cm}$.

Conclusion: Bilateral orthotopic single-system ureteroceles in an adult male are a rare finding especially when intraoperatively found to be open secondary to traumatic effects of large calculi. Ultrasonic and pneumatic lithotripsy is a safe, appropriate, and effective option for bilateral ureteroceles contributing to a large bladder stone burden. Our report contributes to the growing body of literature on orthotopic bilateral single-system ureteroceles in the adult population with concomitant significant stone burden.
\end{abstract}

Keywords: ureterocele, bladder stone, calculi

\section{Introduction}

$\mathbf{U}$ RETEROCELES ARE RARE congenital anomalies, more commonly observed in the pediatric population unilaterally, and associated with duplex collecting systems. In the adult population, ureteroceles are considerably less common with no universally accepted management guidelines. We present a rare case of bilateral open orthotopic single-system ureteroceles in an adult male with mild right-sided hydroureter and large bladder calculi.

\section{Case Presentation}

The patient is a 35-year-old man with a history of asthma who presented to the emergency department with a chief complaint of gross hematuria. He had experienced multiple episodes of gross hematuria alongside moderate right flank pain and significant lower urinary tract symptoms, including straining, hesitancy, and urgency for the past 6 months. The patient denied any personal history of stone disease but endorsed a family history of stones with no family history of malignancy. He also denied any recent trauma, fevers, chills, nausea, vomiting, dysuria, or urinary incontinence. Patient was a nonsmoker and denied any other toxic habits or che- mical exposures. He had never had any urologic intervention either as a child or adult. Physical examination was benign with normal abdominal and genitourinary examinations. Urinalysis was positive for few bacteria and few calcium oxalate crystals, negative for leukocyte esterase and nitrites, and positive for red blood cells. Preoperative urine culture was negative although patient was started on an empiric course of ciprofloxacin. Patient's preoperative creatinine was stable at $0.78 \mathrm{mg} / \mathrm{dL}$, with normal chemistry and complete blood count. CT of the abdomen and pelvis with and without intravenous and per oral contrast demonstrated delayed contrast excretion into the right collecting system with mild hydroureteronephrosis of the right kidney to the level of a right ureterocele, as well as a smaller left-sided ureterocele without proximal hydroureteronephrosis. Both ureteroceles were noted to be orthotopic and protruding into the bladder (Fig. 1). A calculus was noted within the right ureterocele along with a $3.8 \mathrm{~cm}$ bladder calculus was also seen on CT (Fig. 2). No ureteral filling defects were seen (Fig. 3). Bilateral single collecting systems were noted.

The patient underwent elective cystoscopy, cystolithotripsy, removal of bladder stones, and bilateral retrograde pyelograms. A $25 \mathrm{~F}$ sheath was inserted to perform cystoscopy.

\footnotetext{
${ }^{1}$ Department of Urology, Albert Einstein College of Medicine, Bronx, New York, USA.

${ }^{2}$ Department of Urology, NYU Langone Medical Center, New York, New York, USA.
} 


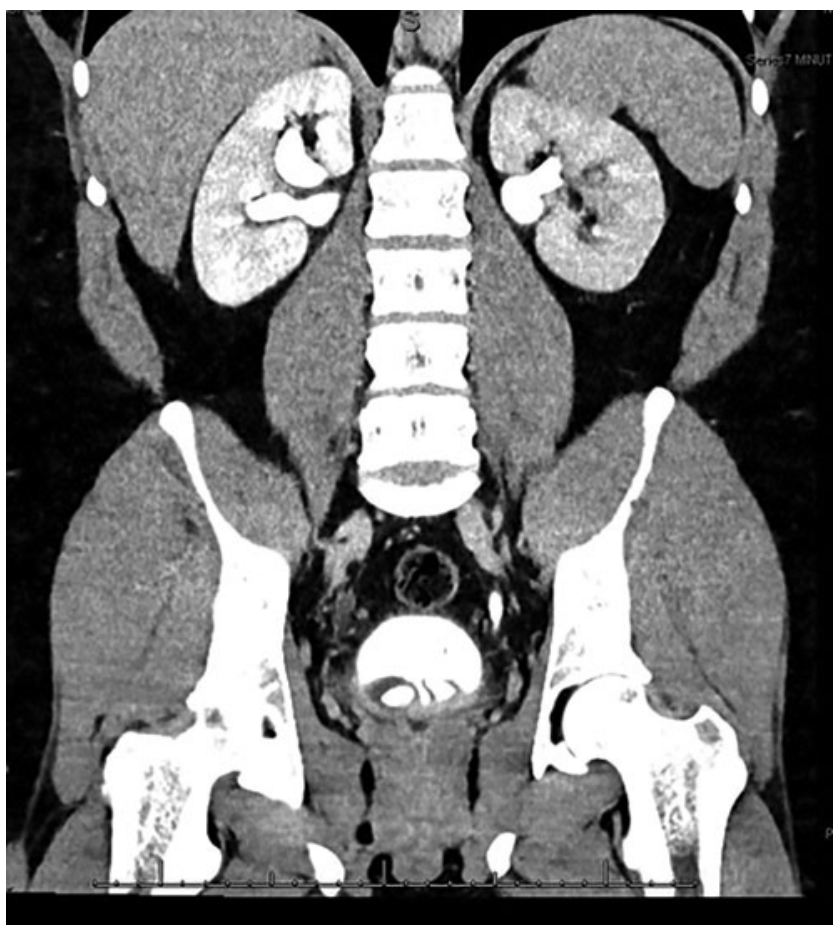

FIG. 1. Cross-sectional CT imaging of the abdomen and pelvis, dated August 2019. The scan reveals bilateral orthotopic ureteroceles, right larger than left, on a coronal view.

Urethroscopy was unremarkable and prostate was found to be small and nonocclusive. Cystoscopy demonstrated bilateral open ureteroceles, right larger than left, appearing to have ruptured secondary to the large stone burden observed in the bladder (Fig. 4). Both ureteral orifices were observed to be patent and cannulated, and bilateral retrograde pyelograms were performed, which revealed normal appearing ureters with mild hydronephrosis. The cystoscope was then replaced with a $28 \mathrm{~F}$ nephroscope and the stones were fragmented through ultrasonic and ballistic lithotripsy (Swiss LithoClast ${ }^{\circledR}$ Trilogy; Boston Scientific). All stone fragments were then removed. An $18 \mathrm{~F}$ Foley catheter was left in place. Patient was discharged same day with outpatient catheter removal and effective trial of void 5 days later. Stone composition was later noted to be calcium oxalate monohydrate. At 3 weeks postoperatively, patient was voiding well without gross hematuria. He reported increased strength of stream and complete absence of lower urinary tract symptoms.

\section{Discussion}

Ureteroceles are cystic dilations of the terminal ureter and are congenital anomalies more commonly observed in the pediatric population with an incidence of $1 / 400$ children, of which $\sim 10 \%$ are bilateral. ${ }^{1}$ Incidence rates in the adult population are reported to range from $1 / 500$ to $1 / 4000$, with cases of orthotopic or bilateral ureteroceles and/or those associated with a single collecting system being even more rare. ${ }^{2}$ Ureteroceles can contribute to a variety of symptoms, including characteristic lower urinary tract symptoms, recurrent urinary tract infection, urinary retention secondary to prolapse, renal dysfunction, and stone formation secondary to urinary stasis. Calculi within unilateral ureteroceles are not uncommon in the literature with reported incidence rates of $4 \%$ to $39 \%$. $^{1}$ Only a handful of cases of bilateral ureteroceles with bilateral stones have been described in the literature with varying clinical presentations. To our knowledge, we present the first reported case of bilateral open orthotopic single-system ureteroceles in an adult male with a large concomitant bladder calculi appearing intraoperatively to have ruptured through the thin ureterocele membrane into the bladder.

Management options for symptomatic ureteroceles primarily involve transurethral opening of the protruding cystic
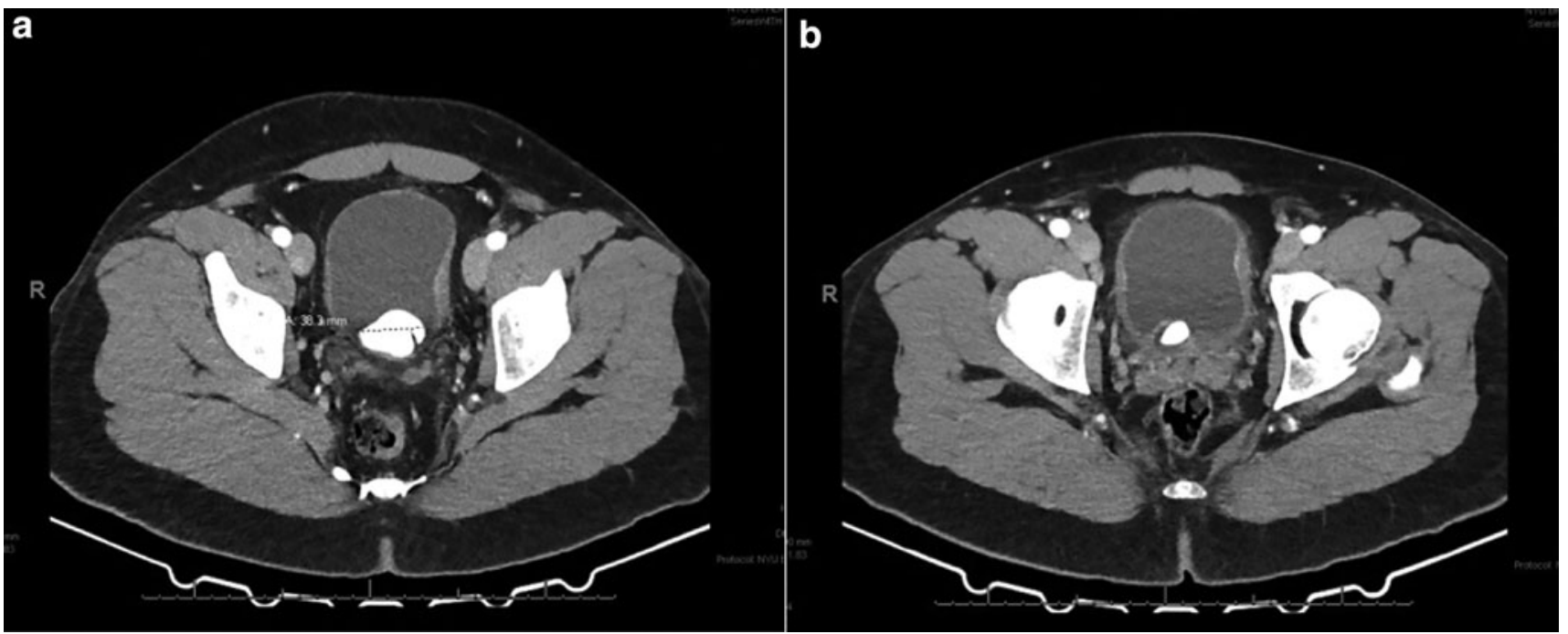

FIG. 2. (a, b) Cross-sectional CT imaging of the abdomen and pelvis, dated August 2019. (a) Reveals a large bladder calculus, measuring $3.8 \mathrm{~cm}$ at its largest dimension, on an axial view. (b) Reveals a large bladder calculus appearing to arise from a right-sided ureterocele on an axial view. 




FIG. 3. Right-sided retrograde pyelogram, dated August 2019. The image reveals a normal-appearing ureter without notable filling defects.

mass, primarily to relieve obstruction or extract stone. One approach commonly cited in the literature involved a low transverse incision using a Collin's knife in a "smiling", manner aimed at opening the ureterocele and leaving behind a decompressed ureterocele flap valve serving as an antireflux mechanism. ${ }^{3}$ Holmium and potassium titanyl phosphate lasers have also been described in the literature to perform a similar endoscopic incision with similarly low rates of vesicoureteral reflux. ${ }^{4}$ Another management option commonly employed is more applicable to severely pro-

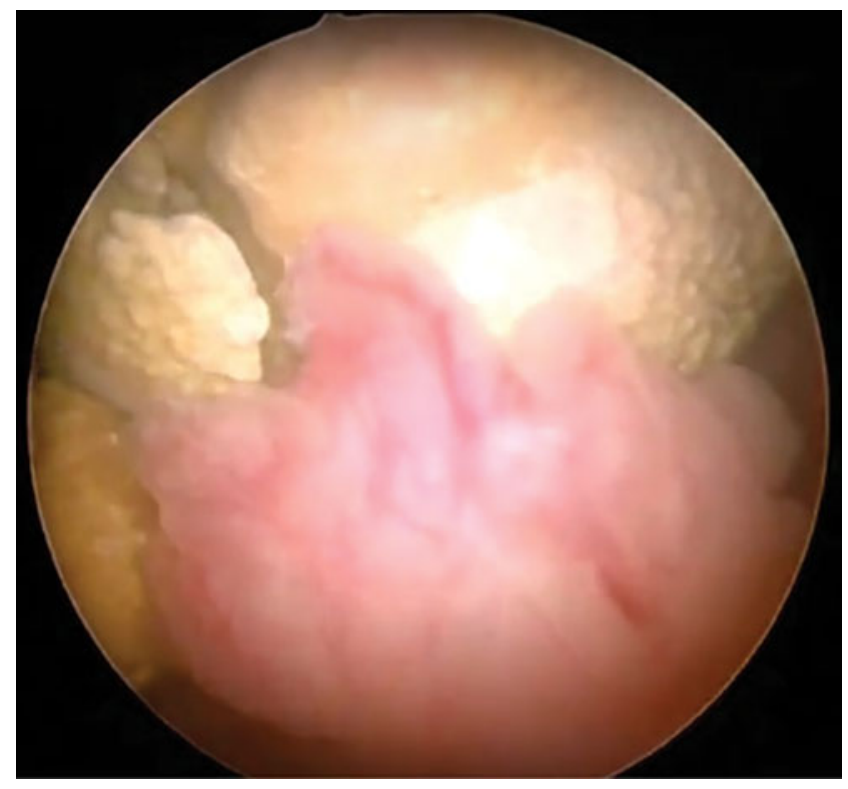

FIG. 4. Cystoscopic view of ureterocele wall defect open from evacuated bladder stone. lapsed ectopic ureteroceles is endoscopic unroofing. A low transverse incision on such ureteroceles leaves excess residual tissue that can continue to prolapse and cause urinary obstruction and thus such patients may need future operations. Endoscopic unroofing can be performed through a variety of instruments, including cold knife, Bugbee electrode, lasers, or scissors. ${ }^{4}$ Extraction of intraureterocele stones can then ensue once the ureterocele is opened regardless of method.

In our case the patient's ureteroceles at the time of cystoscopy were observed to already be opened traumatically from apparent large stone burden. This is supported by preoperative imaging demonstrating large bladder calculi appearing to be arising from the right-sided ureterocele. This case report contributes new and valuable information to the paucity of literature regarding the safe and effective management of adult patients with bilateral ureteroceles and concomitant calculi. In our case, incision and opening of the ureteroceles was deemed unnecessary given that they were found open intraoperatively with spontaneous evacuation of stone. Ultrasonic and pneumatic lithotripsy is a safe, appropriate, and effective option for bilateral ureteroceles contributing to a large bladder stone burden.

\section{Conclusion}

Bilateral orthotopic single-system ureteroceles in an adult male are a rare finding especially when intraoperatively found to be open secondary to traumatic effects of large calculi. Our report contributes to the growing body of literature on orthotopic bilateral single-system ureteroceles in the adult population with concomitant significant stone burden. Further cases of similar presentation must be evaluated to determine best practices for management of bilateral stonecontaining ureteroceles.

\section{Consent}

Written informed consent was obtained from the patient for publication of this case report and accompanying images. A copy of the written consent is available for review by the editor-in-chief of this journal on request.

\section{Disclosure Statement}

No competing financial interests exist.

\section{Funding Information}

No funding was received for this article.

\section{References}

1. Rompré-Brodeur A, Andonian S. Adult bilateral ureteroceles presenting with lower urinary tract symptoms and acute urinary retention. Case Rep Urol 2018;2018:1-5.

2. Peter CA, Schlussel RN, Mendelsohn CL Ectopic ureter, ureterocele, and ureteral anomalies. In: Wein AJ, Partin AW, Peters CA, eds. Campbell-Walsh Urology. Philadelphia, PA: Elsevier, 2016, pp. 3075-3101.

3. Gupta R, Gupta S, Khan D, Basu S. Bilateral single system orthotopic ureterocele with bilateral multiple calculi presented with retention of urine-An urological emergency. J Clin Diagn Res 2017;11:1-2. 
4. Nast KJ, Marietti-Shepherd S, Kaplan G. Scissor ureterocele unroofing endoscopic technique: A novel technique for minimally invasive ureterocele treatment. Videourology 2017;31: DOI: 10.1089/vid.2017.0007.

Address correspondence to:

Ari P. Bernstein, BA

Department of Urology

Albert Einstein College of Medicine

1925 Eastchester Road, Apartment 23G

Bronx, NY 10461

USA

E-mail: ari.bernstein@einsteinmed.org

\begin{tabular}{|c|}
\hline $\begin{array}{c}\text { Abbreviation Used } \\
\mathrm{CT}=\text { computed tomography }\end{array}$ \\
\hline
\end{tabular}

Cite this article as: Bernstein AP, Schulster M, Chao B, Silva MV (2020) Bilateral open ureteroceles with concomitant bladder calculi in an adult male, Journal of Endourology Case Reports 6:3, 103-106, DOI: 10.1089/cren.2019.0142. 